\title{
Fuzzy Adaptive Prescribed Performance Control for Uncertain Horizontal Platform System with Unknown Control Gain
}

\author{
Wei Xiang, Yeguo Sun, and Chunzhi Yang \\ Department of Mathematics, Huainan Normal University, Huainan 232038, China \\ Correspondence should be addressed to Wei Xiang; xiangwei27@126.com
}

Received 11 August 2015; Revised 7 November 2015; Accepted 10 November 2015

Academic Editor: Yang Tang

Copyright (C) 2015 Wei Xiang et al. This is an open access article distributed under the Creative Commons Attribution License, which permits unrestricted use, distribution, and reproduction in any medium, provided the original work is properly cited.

\begin{abstract}
This paper proposes a fuzzy adaptive control method for uncertain horizontal platform system with unknown control gain, which is capable of guaranteeing the prescribed performance bounds. An error transformation is introduced to transform the original constrained system into an equivalent unconstrained one. Then, based on the error transformation technique and the predefined performance technique, a fuzzy adaptive controller is designed for the unconstrained system. It is shown that all the variables of the resulting closed-loop system are bounded. Finally, an illustrative example is given to demonstrate the effectiveness and usefulness of the proposed method.
\end{abstract}

\section{Introduction}

Over the past two decades, it has been acknowledged that several mechanical systems exhibit chaotic behavior [1-3]. The horizontal platform system (HPS) is one of the most interesting and attractive nonlinear dynamical systems. It is a mechanical device that can freely rotate around the horizontal axis. The horizontal platform devices are widely used in offshore and earthquake engineering. Recent research has found that these systems display many dynamic behaviors including chaotic. So, how to suppress chaotic phenomenon for horizontal platform systems (HPSs) is a hot research topic. Until now, a wide variety of approaches have been proposed for HPSs. For example, Wu et al. [4] have applied the Lyapunov direct method to achieve a sufficient criterion for chaos synchronization between two identical HPSs which have been coupled by a linear state error feedback controller. In [5], Pai and Chang proposed a fuzzy sliding mode control scheme to achieve generalized projective synchronization (GPS) of two horizontal chaos platform vibration systems with system uncertainty and external disturbance. By using time-delay feedback control, Ding and Han [6] discussed master-slave synchronization for two identical nonautonomous HPSs. Using a linear state error feedback controller, the robust synchronization of the chaotic HPSs with phase difference and parameter mismatches has been studied in [7]. Based on Lyapunov stability theory, Pai and Yau [8] designed an adaptive sliding mode controller such that the controlled HPS state can be driven to a desired orbit. The problem of robust finite-time synchronization of two nonautonomous HPSs is investigated in [9]. Recently, Xiang and Liu [10] proposed an adaptive fuzzy terminal sliding mode control scheme for uncertain HPS. Based on fuzzy system rules, the proposed control approach guarantees the boundedness of all the signals in closed-loop system. These considered horizontal platform systems are in the form $\dot{x}_{1}=x_{2}, \dot{x}_{2}=$ $f(t, x)+g(t, x) u$, where $x=\left[x_{1}, x_{2}\right]^{T}$ and $g(t, x)=1$. If control gain $g(t, x)$ is unknown, how can we design the control scheme to achieve the stability for uncertain HPS?

To the author's best knowledge, there are few literatures to research the stability problem for uncertain HPS with unknown control gain. To handle unknown nonlinear function $g(t, x)$ in HPS, several nonlinear approximators, for example, fuzzy logic systems and neural networks, have been used. Two of the main features of adaptive fuzzy approaches are as follows: (i) they can be used to deal with those nonlinear systems without satisfying the matching conditions and (ii) they do not require the unknown nonlinear functions 
being linearly parameterized [11-15]. In order to meet control objectives, the used controller in indirect adaptive schemes is in the form $u=\widehat{g}^{-1}\left(x, \theta_{g}\right)(v-f(t, x))$, where $v$ is a new control input. $\hat{g}\left(x, \theta_{g}\right)$ is parameterized approximation of actual function $g(t, x) . \theta_{g}$ represents the adjustable parameter of the approximation. Since the approximation $\widehat{g}\left(x, \theta_{g}\right)$ is generated online by estimating the parameter $\theta_{g}$, one can notice that the above controller is not well-defined because of the singular problem of the controller. In this paper, we use the regularized inverse of $\hat{g}^{-1}\left(x, \theta_{g}\right)$ as $\widehat{g}\left(x, \theta_{g}\right)(\epsilon+$ $\left.\widehat{g}^{2}\left(x, \theta_{g}\right)\right)^{-1}$. The regularized inverse is well-defined even when $\widehat{g}\left(x, \theta_{g}\right)$ is singular. Compared with related works, there are four main contributions that are worth being emphasized:

(1) Compared with the results in [4-10], the uncertain HPS with unknown control gain is considered.

(2) The prescribed performance function (PPF) is incorporated into the control design.

(3) Adaptive laws are proposed to update the fuzzy parameters.

(4) The controller will not appear as singular problem.

Recently, an attempt to establish a priori specified performance control paradigm has been exploited [16], where the maximum overshoot, the convergence rate, and steady-state error are all addressed. Motivated by the above discussion, we will propose an adaptive fuzzy control for HPSs with prescribed transient and steady-state tracking performance. Inspired by $[17,18]$, an improved prescribed performance function (PPF) is incorporated into the control design. An error transformed system is derived by applying the PPF on the original system. Consequently, the tracking error of the original system can be guaranteed within the prescribed bound provided the transformed system is stable. For this purpose, an adaptive prescribed performance control (APPC) is designed for uncertain HPS in the presence of system uncertainties and external disturbance, which also allows proving the closed-loop stability. A comparative example is given to emphasize the effectiveness of the proposed APPC based on the PPF design.

The organization of this paper is described as follows. In the next section, system model is derived, and the assumptions are also given. In Section 3, the design of the proposed control strategies is discussed. The simulation results are presented to demonstrate the effectiveness of proposed control scheme in Section 4. Conclusion is presented in Section 5.

\section{System Descriptions and Problem Formulations}

The HPS is a mechanical device composed of a platform and an accelerometer located on the platform (see Figure 1). The platform can freely rotate about the horizontal axis, which penetrates its mass center. The accelerometer produces an output signal to the actuator, subsequently generating a torque to inverse the rotation of the platform to balance

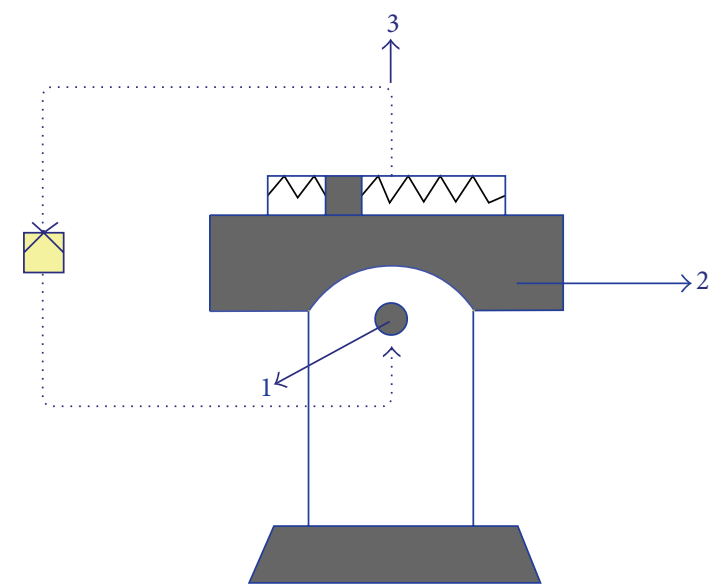

FIgure 1: Physical model of the horizontal platform system (1).

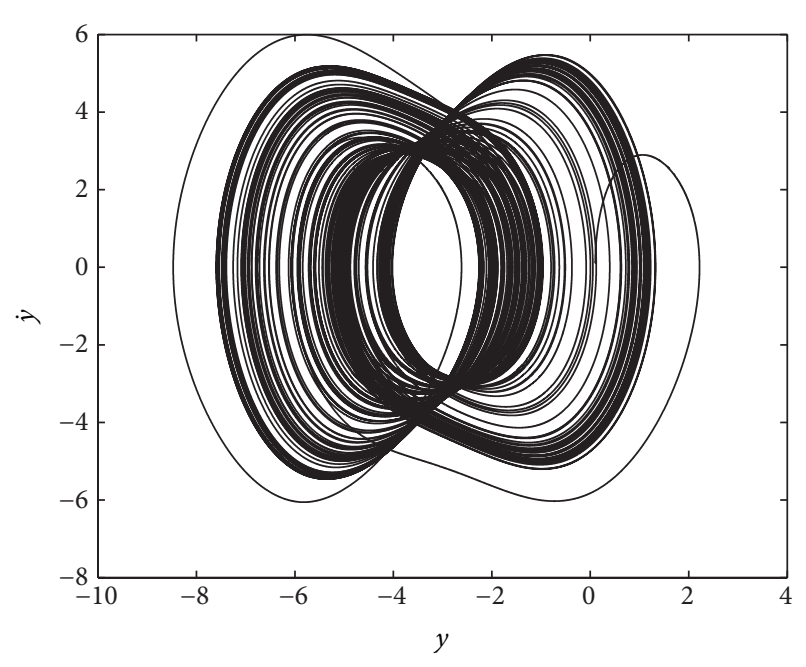

FIGURE 2: Phase plane trajectory of the horizontal platform system (1).

the HPS, when the platform deviates from horizon. The motion equations of the HPS are given by $[9,10]$

$$
\begin{aligned}
A \ddot{y} & +D \dot{y}+k g \sin y-\frac{3 g}{R}(B-C) \cos y \sin y \\
& =F \cos \omega t,
\end{aligned}
$$

where $y$ is the rotation of the platform relative to horizon, $A, B$, and $C$ are inertia moment of the platform, $D$ is the damping coefficient, $k$ is the proportional constant of the accelerometer, $g$ is the acceleration constant of gravity, $R$ is the radius of Earth, and $F \cos \omega t$ is the harmonic torque. System (1) exhibits chaotic behavior with $A=0.3, B=0.5$, $C=0.2, D=0.4, k=0.11559633, R=6378000, F=3.4$, and $\omega=1.8$ (see Figure 2).

For simplicity, we introduce the following notations: $x=$ $\left[x_{1}, x_{2}\right]^{T}=[y, \dot{y}]^{T}$; then the dynamic model of (1) with 
unknown control gain and external disturbances can be described by the following equations:

$$
\begin{aligned}
& \dot{x}_{1}=x_{2}, \\
& \dot{x}_{2}=f(t, x)+d(t, x)+g(t, x) u(t),
\end{aligned}
$$

where $u(t) \in R$ is the control input, $g(t, x)$ is unknown control gain, and $d(t, x)$ is unknown external disturbance. $f(t, x)=-a x_{2}-b \sin x_{1}+l \cos x_{1} \sin x_{1}+h \cos \omega t$ is assumed to be unknown, $a=D / A, b=k g / A, l=3 g(B-C) / R A$, and $h=F / A$.

The objective of this paper is to construct a fuzzy adaptive controller for system (2) such that

(P1) the system state $y$ tracks the reference signal $y_{d} \in R$ and all the signals in the closed-loop system remain bounded,

(P2) both prescribed transient and steady-state behavioral bounds on the tracking error $e=y-y_{d}=x_{1}-y_{d}$ are achieved.

To meet the objective, the following assumptions are made for the system (2).

Assumption 1. The state vector $x$ is measurable, and the references $y_{d}, \dot{y}_{d}$, and $\ddot{y}_{d}$ are continuous and bounded.

Assumption 2. $f(t, x)$ and $g(t, x)$ are unknown but bounded. And there exists $\delta>0$ such that $g(t, x)>\delta$.

Remark 3. The present control scheme in this paper can guarantee control objectives without the knowledge of the $\delta$ value in Assumption 2.

\subsection{Prescribed Performance}

Definition 4. A smooth function $\mu(t): R^{+} \rightarrow R^{+}$is called a prescribed performance function (PPF) if $\mu(t)$ is decreasing and $\lim _{t \rightarrow \infty} \mu(t)=\mu_{\infty}$.

In this paper, we select $\mu(t)$ as

$$
\mu(t)=\left(\mu_{0}-\mu_{\infty}\right) e^{-\kappa t}+\mu_{\infty},
$$

where $\mu_{0}>\mu_{\infty}$ and $\kappa>0$ are design parameters.

It is sufficient to achieve the control objective (P2) if condition (4) holds

$$
-\delta_{\min } \mu(t)<e(t)<\delta_{\max } \mu(t), \quad \forall t \geq 0,
$$

where $\delta_{\min }$ and $\delta_{\max }$ are design constants.

Remark 5. The transient and steady-state performances can be designed a priori by tuning the parameters $\delta_{\min }, \delta_{\max }, \mu_{0}$, $\kappa, \mu_{\infty}$.

To represent (4) by an equality form, we employ an error transformation as

$$
e(t)=\mu(t) s(z)
$$

where $z$ is the transformed error and $s(\cdot)$ is smooth, strictly increasing function and satisfies the following condition:

$$
\begin{aligned}
-\delta_{\min } & <s(z)<\delta_{\max }, \quad \forall z \in L_{\infty}, \\
\lim _{z \rightarrow-\infty} s(z) & =-\delta_{\min } \\
\lim _{z \rightarrow+\infty} s(z) & =\delta_{\max } .
\end{aligned}
$$

Note that $s(z)$ are strictly increasing functions; we have

$$
z=s^{-1}\left(\frac{e(t)}{\mu(t)}\right) \text {. }
$$

Note that, for any initial condition $e(0)$, if parameters $\mu_{0}$, $\delta_{\min }$, and $\delta_{\max }$ are selected such that $-\delta_{\min } \mu(0)<e(0)<$ $\delta_{\max } \mu(0)$ and $z$ can be controlled to be bounded, then $-\delta_{\min }<s(z)<\delta_{\max }$ holds. Thus, the condition $-\delta_{\min } \mu(t)<$ $e(t)<\delta_{\max } \mu(t)$ can be guaranteed. Now, the tracking control problem of system (2) is now transformed to stabilize the transformed system (7).

Differentiating (7) with respect to time $t$ yields

$$
\dot{z}=\frac{\partial s^{-1}}{\partial(e(t) / \mu(t))} \frac{1}{\mu(t)}\left[x_{2}-\dot{y}_{d}-\frac{e(t) \dot{\mu}(t)}{\mu(t)}\right] .
$$

Let $0<r=\left(\partial s^{-1} / \partial(e(t) / \mu(t))\right)(1 / \mu(t))<r_{m}$, where $r_{m}$ is a positive constant. Then (8) can be rewritten as

$$
\dot{z}=r\left[x_{2}-\dot{y}_{d}-\frac{e \dot{\mu}}{\mu}\right] .
$$

Moreover, we obtain

$$
\ddot{z}=r F_{1}(x, \mu, e)+r F_{2}(t, x)+r g(t, x) u,
$$

where $F_{1}(x, \mu, e)=(\dot{r} / r)\left(x_{2}-\dot{y}_{d}-e \dot{\mu} / \mu\right)-\left(\ddot{y}_{d}+\dot{e} \dot{\mu} / \mu+\right.$ $\left.e \ddot{\mu} \mu / \mu^{2}-e \dot{\mu}^{2} / \mu^{2}\right)$ is known nonlinear function and $F_{2}(t, x)=$ $f(t, x)+d(t, x)$ is a nonlinear function including unknown dynamics and disturbances.

Remark 6. In general, $s(z)$ is chosen as $s(z)=\left(\delta_{\max } e^{z}-\right.$ $\left.\delta_{\min } e^{-z}\right) /\left(e^{z}+e^{-z}\right)$. So, we can calculate that $r=\left(1 /\left(\lambda+\delta_{\min }\right)-\right.$ $\left.1 /\left(\lambda-\delta_{\max }\right)\right) / 2 \mu$ such that $0<r<\left(\delta_{\max }+\delta_{\min }\right) / \mu_{\infty} \delta_{\max } \delta_{\min }$, where $\lambda=e / \mu$.

2.2. Fuzzy Logic Systems. The basic configuration of a fuzzy logic system consists of a fuzzifier, some fuzzy IF-THEN rules, a fuzzy inference engine, and a defuzzifier. The fuzzy inference engine uses the fuzzy IF-THEN rules to perform a mapping from an input vector $x=\left[x_{1}, x_{2}, \ldots, x_{n}\right]^{T} \in R^{n}$ to an output $\alpha(x) \in R$. The $i$ th fuzzy rule is written as

$$
\begin{aligned}
& \text { Rule } i \text { : if } x_{1} \text { is } F_{1}^{i} \text { and } \ldots \text { and } x_{n} \text { is } F_{n}^{i} \text { then } \alpha(x) \\
& \text { is } \alpha_{i} \text {, }
\end{aligned}
$$

where $F_{1}^{i}, F_{2}^{i}, \ldots$ and $F_{n}^{i}$ are fuzzy sets and $\alpha_{i}$ is the fuzzy singleton for the output in the $i$ th rule. By using the singleton fuzzifier, product inference, and the center-average 
defuzzifier, the output of the fuzzy system can be expressed as follows:

$$
\alpha(x)=\frac{\sum_{j=1}^{N} \alpha_{j} \prod_{i=1}^{n} \mu_{F_{i}^{j}}\left(x_{i}\right)}{\sum_{j=1}^{N}\left[\prod_{i=1}^{n} \mu_{F_{i}^{j}}\left(x_{i}\right)\right]}=\theta^{T} \psi(x),
$$

where $\mu_{F_{i}^{j}}\left(x_{i}\right)$ is the degree of membership of $x_{i}$ to $F_{i}^{j}, N$ is the number of fuzzy rules, $\theta=\left[\alpha_{1}, \ldots, \alpha_{N}\right]^{T}$ is the adjustable parameter vector, and $\psi(x)=\left[p_{1}(x), p_{2}(x), \ldots, p_{N}(x)\right]^{T}$, where

$$
p_{j}(x)=\frac{\prod_{i=1}^{n} \mu_{F_{i}^{j}}\left(x_{i}\right)}{\sum_{j=1}^{N}\left[\prod_{i=1}^{n} \mu_{F_{i}^{j}}\left(x_{i}\right)\right]}
$$

is the fuzzy basis function. It is assumed that fuzzy basis functions are selected so that there is always at least one active rule.

\section{Main Results}

Define the filtered error as

$$
\sigma=\imath z+\dot{z}
$$

where $\iota>0$ is a positive constant such that the tracking error $z$ is bounded as long as $\sigma$ is bounded.

Due to the fact that $F_{2}(t, x)$ and $g(t, x)$ are unknown, we need to use fuzzy logic system to approximate the nonlinear unknown functions. By applying the introduced fuzzy systems, approximation of function $F_{2}(t, x)$ and $g(t, x)$ can be expressed as follows:

$$
\begin{gathered}
\widehat{F}_{2}\left(x, \theta_{F_{2}}\right)=\theta_{F_{2}}^{T} \psi_{F_{2}}(x), \\
\widehat{g}\left(x, \theta_{g}\right)=\theta_{g}^{T} \psi_{g}(x) .
\end{gathered}
$$

Optimal parameters $\theta_{F_{2}}^{*}$ and $\theta_{g}^{*}$ can be defined such that

$$
\begin{aligned}
\theta_{F_{2}}^{*} & =\arg \min _{\theta}\left[\sup \left|F_{2}(t, x)-\widehat{F}_{2}\left(x, \theta_{F_{2}}\right)\right|\right], \\
\theta_{g}^{*} & =\arg \min _{\theta}\left[\sup \left|g(t, x)-\widehat{g}\left(x, \theta_{g}\right)\right|\right] .
\end{aligned}
$$

Define the parameter estimation errors and the fuzzy approximation errors as follows:

$$
\begin{gathered}
\tilde{\theta}_{F_{2}}=\theta_{F_{2}}-\theta_{F_{2}}^{*}, \\
\tilde{\theta}_{g}=\theta_{g}-\theta_{g}^{*}, \\
\varepsilon_{F_{2}}(x)=F_{2}\left(x, \theta_{F_{2}}^{*}\right)-F_{2}(t, x), \\
\varepsilon_{g}(x)=g\left(x, \theta_{g}^{*}\right)-g(t, x) .
\end{gathered}
$$

We assume that $\left|\varepsilon_{F_{2}}\right| \leq \varepsilon_{1}$ and $\left|\varepsilon_{g}\right| \leq \varepsilon_{2}$, where $\varepsilon_{1}$ and $\varepsilon_{2}$ are positive constants.
The adaptive prescribed performance controller can be specified as

$$
u=u_{1}+u_{2}
$$

where

$$
\begin{gathered}
u_{1}=-\hat{g}\left(x, \theta_{g}\right)\left[\epsilon+\widehat{g}^{2}\left(x, \theta_{g}\right)\right]^{-1} \\
\cdot\left[F_{3}(x, \mu, e)+\widehat{F}_{2}\left(x, \theta_{F_{2}}\right)+\eta_{1} \sigma\right], \\
u_{2}=-\frac{\left(\varepsilon_{1}+\varepsilon_{2}\left|u_{1}\right|+\left|u_{3}\right|\right) \cdot \operatorname{sign}(\sigma)}{\delta},
\end{gathered}
$$

where $\epsilon$ is a small positive constant, $\eta_{1}$ is a designed positive constant, $u_{3}=\epsilon\left[\epsilon+\widehat{g}^{2}\left(x, \theta_{g}\right)\right]^{-1}\left[F_{3}(x, \mu, e)+\widehat{F}_{2}\left(x, \theta_{F_{2}}\right)+\eta_{1} \sigma\right]$, $F_{3}(x, \mu, e)=F_{1}(x, \mu, e)+\iota\left(x_{2}-\dot{y}_{d}-e \dot{\mu} / \mu\right)$, and $\delta$ is assumed to be known.

To generate the approximations $F_{2}(t, x)$ and $g(t, x)$ online, we choose the following adaptive laws:

$$
\begin{aligned}
& \dot{\theta}_{F_{2}}=r \varsigma_{1} \sigma \psi_{F_{2}}, \\
& \dot{\theta}_{g}=r \varsigma_{2} u_{1} \sigma \psi_{g},
\end{aligned}
$$

where $\varsigma_{1}, \varsigma_{2}$ are positive constants.

So, we have the following result.

Theorem 7. Consider the controlled HPS (2) and the error transform (7). Suppose that Assumptions 1 and 2 are satisfied. Then controller (18) with the adaptive laws given by (21) can guarantee that all signals in the closed-loop system are bounded. Furthermore, The prescribed control performance (4) is preserved.

Proof. Consider a Lyapunov function as

$$
V=\frac{1}{2}\left[\sigma^{2}+\frac{\widetilde{\theta}_{F_{2}}^{T} \widetilde{\theta}_{F_{2}}}{\varsigma_{1}}+\frac{\widetilde{\theta}_{g}^{T} \widetilde{\theta}_{g}}{\varsigma_{2}}\right] .
$$

The time derivative of $V$ is given by

$$
\begin{aligned}
\dot{V} & =\sigma[\iota \dot{z}+\ddot{z}]+\frac{\widetilde{\theta}_{F_{2}}^{T} \dot{\tilde{\theta}}_{F_{2}}}{\varsigma_{1}}+\frac{\widetilde{\theta}_{g}^{T} \dot{\tilde{\theta}}_{g}}{\varsigma_{2}} \\
& =\sigma\left(r l\left(x_{2}-\dot{y}_{d}-\frac{e \dot{\mu}}{\mu}\right)+r F_{1}(x, \mu, e)+r F_{2}(t, x)\right. \\
& +r g(t, x) u)+\frac{\widetilde{\theta}_{F_{2}}^{T} \dot{\theta}_{F_{2}}}{\varsigma_{1}}+\frac{\widetilde{\theta}_{g}^{T} \dot{\theta}_{g}}{\varsigma_{2}}=\sigma\left(r F_{3}(x, \mu, e)\right. \\
& +r\left(F_{2}^{*}\left(x, \theta_{F_{2}}^{*}\right)-\varepsilon_{F_{2}}\right)+r\left(g(t, x)-\widehat{g}\left(x, \theta_{g}\right)\right) u_{1} \\
& \left.+r \hat{g}\left(x, \theta_{g}\right) u_{1}+r g(t, x) u_{2}\right)+\frac{\widetilde{\theta}_{F_{2}}^{T} \dot{\theta}_{F_{2}}}{\varsigma_{1}}+\frac{\widetilde{\theta}_{g}^{T} \dot{\theta}_{g}}{\varsigma_{2}} .
\end{aligned}
$$


Notice that $\hat{g}^{2}\left(x, \theta_{g}\right)\left[\epsilon+\hat{g}^{2}\left(x, \theta_{g}\right)\right]^{-1}=1-\epsilon[\epsilon+$ $\left.\hat{g}^{2}\left(x, \theta_{g}\right)\right]^{-1}$. One can obtain

$$
\begin{aligned}
\dot{V} & =\sigma\left(-r \eta_{1} \sigma+r \widetilde{\theta}_{F_{2}}^{T} \psi_{F_{2}}-r \varepsilon_{F_{2}}+r \widetilde{\theta}_{g}^{T} \psi_{g} u_{1}-r \varepsilon_{g} u_{1}\right. \\
& \left.+r u_{3}+r g(t, x) u_{2}\right)+\frac{\widetilde{\theta}_{F_{2}}^{T} \dot{\theta}_{F_{2}}}{\varsigma_{1}}+\frac{\widetilde{\theta}_{g}^{T} \dot{\theta}_{g}}{\varsigma_{2}} \leq-r \eta_{1} \sigma^{2} \\
& +r \sigma \widetilde{\theta}_{F_{2}}^{T} \psi_{F_{2}}+r \varepsilon_{1}|\sigma|+r \sigma \widetilde{\theta}_{g}^{T} \psi_{g} u_{1}+r \varepsilon_{2}|\sigma|\left|u_{1}\right| \\
& +r|\sigma|\left|u_{3}\right|+r \sigma g(t, x) u_{2}+\frac{\widetilde{\theta}_{F_{2}}^{T} \dot{\theta}_{F_{2}}}{\varsigma_{1}}+\frac{\widetilde{\theta}_{g}^{T} \dot{\theta}_{g}}{\varsigma_{2}} .
\end{aligned}
$$

According to Assumption 2 and (20), we have $\sigma g(t, x) u_{2}<-L|\sigma|$, where $L=\varepsilon_{1}+\varepsilon_{2}\left|u_{1}\right|+\left|u_{3}\right|$. So, by using adaptation laws (21), we obtain

$$
\dot{V} \leq-r \eta_{1} \sigma^{2}
$$

Therefore, $\dot{V}$ is negative semidefinite and $V \in L_{\infty}$, which implies that $z, \widetilde{\theta}_{F_{2}}$, and $\widetilde{\theta}_{g}$ are bounded. So, $z \in L_{\infty}$. Then, according to the properties of function $s(z)$, we know that $-\delta_{\min }<s(z)<\delta_{\max }$. Then, one can conclude that tracking control of system (2) with prescribed error performance (4) is achieved. This completes the proof.

Remark 8. Compared with the results in [7-10], the unknown control gain is considered in this paper.

Remark 9. In order to avoid the chatter in controller (18), we can modify $u_{2}$ as follows:

$$
u_{2}=-\frac{\sigma|\sigma|\left(\varepsilon_{1}+\varepsilon_{2}\left|u_{1}\right|+\left|u_{3}\right|\right)}{\delta|\sigma|^{2}+\xi}
$$

where $\xi$ is a design time-varying parameter defined as $\dot{\xi}=$ $-\eta_{2}|\sigma|\left(\varepsilon_{1}+\varepsilon_{2}\left|u_{1}\right|+\left|u_{3}\right|\right) /\left(\delta|\sigma|^{2}+\xi\right)$, where $\eta_{2}$ is a positive constant.

Remark 10. The control law (18) can effectively avoid the singularity problem, because even when $\widehat{g}\left(x, \theta_{g}\right)$ is singular, this controller is well-defined.

In order to obtain a control law with its adaptive laws guaranteeing control objectives without the knowledge of the $\delta$ value (see Assumption 2) and the reconstruction error bounds $\varepsilon_{1}$ and $\varepsilon_{2}$ in the controller, we will propose a new adaptive control scheme.
Theorem 11. Consider the controlled HPS (2) and the error transform (7), and suppose that Assumptions 1 and 2 are satisfied. Consider the control law

$$
u=\bar{u}_{1}+\bar{u}_{2}
$$

where

$$
\begin{aligned}
& \bar{u}_{1}=-\widehat{g}\left(x, \theta_{g}\right)\left[\epsilon+\widehat{g}^{2}\left(x, \theta_{g}\right)\right]^{-1} \\
& \cdot\left[F_{3}(x, \mu, e)+\widehat{F}_{2}\left(x, \theta_{F_{2}}\right)+\eta_{1} \sigma\right], \\
& \bar{u}_{2}=-\frac{\sigma|\sigma|\left(\widehat{\varepsilon}_{F}+\widehat{\varepsilon}_{g}\left|u_{c}\right|+\widehat{\varepsilon}_{\delta}\left|\bar{u}_{3}\right|\right)}{|\sigma|^{2}+\xi},
\end{aligned}
$$

where $\bar{u}_{3}=\epsilon\left[\epsilon+\widehat{g}^{2}\left(x, \theta_{g}\right)\right]^{-1}\left[F_{3}(x, \mu, e)+\widehat{F}_{2}\left(x, \theta_{F_{2}}\right)+\eta_{1} \sigma\right]$. And the adaptive laws are given as

$$
\begin{aligned}
& \dot{\theta}_{F_{2}}=r \varsigma_{1} \sigma \psi_{F_{2}}, \\
& \dot{\theta}_{g}=r \varsigma_{2} \bar{u}_{1} \sigma \psi_{g}, \\
& \dot{\widehat{\varepsilon}}_{F}=\eta_{2} r|\sigma|, \\
& \dot{\widehat{\varepsilon}}_{g}=\eta_{3} r|\sigma|\left|\bar{u}_{1}\right|, \\
& \dot{\widehat{\varepsilon}}_{\delta}=\eta_{4} r|\sigma|\left|\bar{u}_{3}\right|, \\
& \dot{\xi}=-\frac{\eta_{5}|\sigma|\left(\widehat{\varepsilon}_{F}+\widehat{\varepsilon}_{g}\left|\bar{u}_{1}\right|+\widehat{\varepsilon}_{\delta}\left|\bar{u}_{3}\right|\right)}{|\sigma|^{2}+\xi},
\end{aligned}
$$

where $\widehat{\varepsilon}_{F}, \widehat{\varepsilon}_{g}$, and $\widehat{\varepsilon}_{\delta}$ are the estimates of $\varepsilon_{F}^{*}=\varepsilon_{1} / \delta, \varepsilon_{g}^{*}=\varepsilon_{2} / \delta$, and $\varepsilon_{\delta}^{*}=1 / \delta$, respectively. Suppose $\varsigma_{1}>0, \varsigma_{2}>0, \eta_{i}>0$, $i=1,2,3,4,5, \xi(0)>0$, and all signals of the overall closedloop system are bounded and the tracking errors remain in a neighborhood of the origin within the prescribed performance bounds for all $t \geq 0$.

Proof. Consider a Lyapunov function as

$$
\begin{aligned}
V & =\frac{1}{2}\left[\sigma^{2}+\frac{\widetilde{\theta}_{F_{2}}^{T} \widetilde{\theta}_{F_{2}}}{\varsigma_{1}}+\frac{\widetilde{\theta}_{g}^{T} \widetilde{\theta}_{g}}{\varsigma_{2}}+\frac{\delta}{\eta_{2}} \widetilde{\varepsilon}_{F}^{2}+\frac{\delta}{\eta_{3}} \widetilde{\varepsilon}_{g}^{2}+\frac{\delta}{\eta_{4}} \widetilde{\varepsilon}_{\delta}^{2}\right. \\
& \left.+\frac{\delta}{\eta_{5}} \xi^{2}\right],
\end{aligned}
$$

where $\widetilde{\varepsilon}_{F}=\varepsilon_{F}^{*}-\widehat{\varepsilon}_{F}, \widetilde{\varepsilon}_{g}=\varepsilon_{g}^{*}-\widehat{\varepsilon}_{g}$, and $\widetilde{\varepsilon}_{\delta}=\varepsilon_{\delta}^{*}-\widehat{\varepsilon}_{\delta}$. 
The time derivative of $V$ is given by

$$
\begin{aligned}
& \dot{V}=\sigma[\iota \dot{z}+\ddot{z}]+\frac{\widetilde{\theta}_{F_{2}}^{T} \dot{\tilde{\theta}}_{F_{2}}}{\varsigma_{1}}+\frac{\widetilde{\theta}_{g}^{T} \dot{\tilde{\theta}}_{g}}{\varsigma_{2}}-\frac{\delta}{\eta_{2}} \widetilde{\varepsilon}_{F} \widehat{\varepsilon}_{F}-\frac{\delta}{\eta_{3}} \widetilde{\varepsilon}_{g} \widehat{\varepsilon}_{g} \\
& -\frac{\delta}{\eta_{4}} \widetilde{\varepsilon}_{\delta} \widehat{\varepsilon}_{\delta}+\frac{\delta}{\eta_{5}} \xi \dot{\xi}=\sigma\left(r F_{3}(x, \mu, e)\right. \\
& +r\left(F_{2}^{*}\left(x, \theta_{F_{2}}^{*}\right)-\varepsilon_{F_{2}}\right)+r\left(g(t, x)-\widehat{g}\left(x, \theta_{g}\right)\right) \bar{u}_{1} \\
& \left.+r \hat{g}\left(x, \theta_{g}\right) \bar{u}_{1}+r g(t, x) \bar{u}_{2}\right)+\frac{\widetilde{\theta}_{F_{2}}^{T} \dot{\theta}_{F_{2}}}{\varsigma_{1}}+\frac{\widetilde{\theta}_{g}^{T} \dot{\theta}_{g}}{\varsigma_{2}} \\
& -\frac{\delta}{\eta_{2}} \widetilde{\varepsilon}_{F} \widehat{\varepsilon}_{F}-\frac{\delta}{\eta_{3}} \widetilde{\varepsilon}_{g} \widehat{\varepsilon}_{g}-\frac{\delta}{\eta_{4}} \widetilde{\varepsilon}_{\delta} \widehat{\varepsilon}_{\delta}+\frac{\delta}{\eta_{5}} \xi \dot{\xi}=\sigma\left(-r \eta_{1} \sigma\right. \\
& +r \widetilde{\theta}_{F_{2}}^{T} \psi_{F_{2}}-r \varepsilon_{F_{2}}+r \widetilde{\theta}_{g}^{T} \psi_{g} \bar{u}_{1}-r \varepsilon_{g} \bar{u}_{1}+r \bar{u}_{3} \\
& \left.+r g(t, x) \bar{u}_{2}\right)+\frac{\widetilde{\theta}_{F_{2}}^{T} \dot{\theta}_{F_{2}}}{\varsigma_{1}}+\frac{\widetilde{\theta}_{g}^{T} \dot{\theta}_{g}}{\varsigma_{2}}-\frac{\delta}{\eta_{2}} \widetilde{\varepsilon}_{F} \widehat{\varepsilon}_{F}-\frac{\delta}{\eta_{3}} \\
& \cdot \widetilde{\varepsilon}_{g} \widehat{\varepsilon}_{g}-\frac{\delta}{\eta_{4}} \widetilde{\varepsilon}_{\delta} \widehat{\varepsilon}_{\delta}+\frac{\delta}{\eta_{5}} \xi \dot{\xi} \leq-r \eta_{1} \sigma^{2}+r \sigma \widetilde{\theta}_{F_{2}}^{T} \psi_{F_{2}} \\
& +r \varepsilon_{1}|\sigma|+r \sigma \widetilde{\theta}_{g}^{T} \psi_{g} \bar{u}_{1}+r \varepsilon_{2}|\sigma|\left|\bar{u}_{1}\right|+r|\sigma|\left|\bar{u}_{3}\right| \\
& +r \sigma g(t, x) \bar{u}_{2}+\frac{\widetilde{\theta}_{F_{2}}^{T} \dot{\theta}_{F_{2}}}{\varsigma_{1}}+\frac{\widetilde{\theta}_{g}^{T} \dot{\theta}_{g}}{\varsigma_{2}}-\frac{\delta}{\eta_{2}} \widetilde{\varepsilon}_{F} \widehat{\varepsilon}_{F}-\frac{\delta}{\eta_{3}} \\
& \cdot \widetilde{\varepsilon}_{g} \widehat{\varepsilon}_{g}-\frac{\delta}{\eta_{4}} \widetilde{\varepsilon}_{\delta} \widehat{\varepsilon}_{\delta}+\frac{\delta}{\eta_{5}} \xi \dot{\xi} \leq-r \eta_{1} \sigma^{2}+r \sigma \widetilde{\theta}_{F_{2}}^{T} \psi_{F_{2}} \\
& +r \sigma \widetilde{\theta}_{g}^{T} \psi_{g} \bar{u}_{1}+r \delta \varepsilon_{F}^{*}|\sigma|+r \varepsilon_{g}^{*}|\sigma|\left|\bar{u}_{1}\right|+r \delta \varepsilon_{\delta}^{*}|\sigma|\left|\bar{u}_{3}\right| \\
& +r \sigma g(t, x) \bar{u}_{2}+\frac{\widetilde{\theta}_{F_{2}}^{T} \dot{\theta}_{F_{2}}}{\varsigma_{1}}+\frac{\widetilde{\theta}_{g}^{T} \dot{\theta}_{g}}{\varsigma_{2}}-\frac{\delta}{\eta_{2}} \widetilde{\varepsilon}_{F} \widehat{\varepsilon}_{F}-\frac{\delta}{\eta_{3}} \\
& \cdot \widetilde{\varepsilon}_{g} \widehat{\varepsilon}_{g}-\frac{\delta}{\eta_{4}} \widetilde{\varepsilon}_{\delta} \widehat{\varepsilon}_{\delta}+\frac{\delta}{\eta_{5}} \xi \dot{\xi} \text {. }
\end{aligned}
$$

According to Assumption 2 and (29), we have

$$
\begin{aligned}
r \sigma g(t, x) \bar{u}_{2}< & -\frac{r \delta|\sigma|^{2}|\sigma|\left(\widehat{\varepsilon}_{F}+\widehat{\varepsilon}_{g}\left|u_{c}\right|+\widehat{\varepsilon}_{\delta}\left|\bar{u}_{3}\right|\right)}{|\sigma|^{2}+\xi} \\
= & -r|\sigma|\left(\widehat{\varepsilon}_{F}+\widehat{\varepsilon}_{g}\left|u_{c}\right|+\widehat{\varepsilon}_{\delta}\left|\bar{u}_{3}\right|\right) \\
& +\frac{r \xi|\sigma|\left(\widehat{\varepsilon}_{F}+\widehat{\varepsilon}_{g}\left|u_{c}\right|+\widehat{\varepsilon}_{\delta}\left|\bar{u}_{3}\right|\right)}{|\sigma|^{2}+\xi} .
\end{aligned}
$$

$$
\begin{aligned}
& \dot{\hat{\rho}}=\varsigma_{1}|\bar{s}|, \\
& \dot{\theta}_{g}=\varsigma_{2} \overline{\bar{u}}_{1} \bar{s} \psi_{g},
\end{aligned}
$$

where $\hat{\rho}$ is the estimate function of $\rho$. In all the simulation process, the initial values of the chaotic system are 


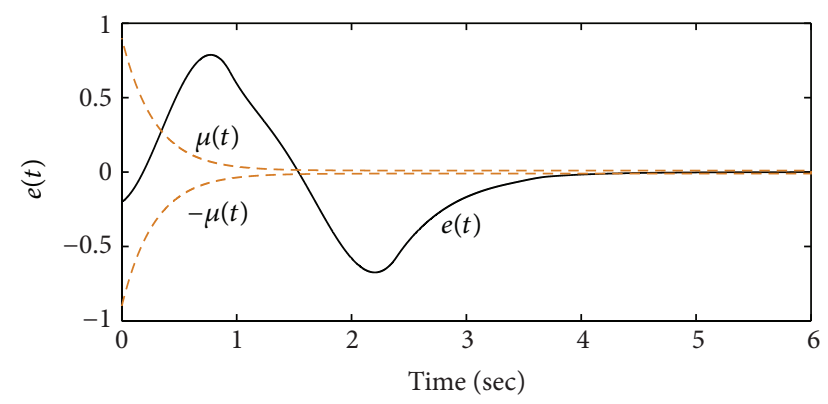

Figure 3: Time response $e(t)$ of error dynamic system (36) under the method of (38).

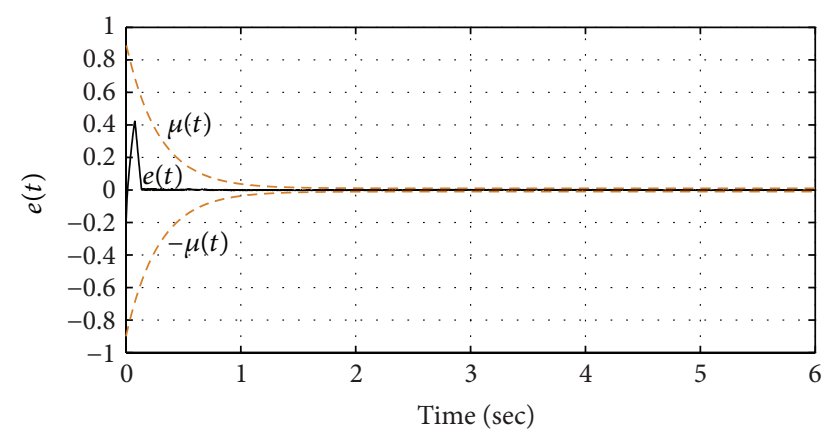

FIgURE 4: Time response $e(t)$ of error dynamic system (36) under the present method of (18).

$\left[x_{1}(0), x_{2}(0)\right]^{T}=[-0.2,0.5]^{T}, y_{d}=0.4 \sin (2 t), d(t, x)=$ $0.5 \sin \left(2 x_{1}\right), g(t, x)=2-\sin \left(x_{1}\right), c_{1}=\varsigma_{1}=\varsigma_{2}=3, \eta=2, A=$ $0.3, B=0.5, C=0.2, D=0.4, k=0.11559633, R=6378000$, $F=3.4, \omega=1.8$, and $\delta=0.5$. We define seven Gaussian membership functions uniformly distributed on the interval $[-10,10]$. And we choose the initial values of parameters of the fuzzy systems as $\theta_{F_{2}}=\theta_{g}=0.1$. Figure 3 shows the time response of the error state $e(t)$ under the control method (38). From Figure 3, we know that the tracking error $e(t)$ violates the prescribed error bounds and cannot achieve the good performances in the beginning stage.

Now, by using the present control scheme (18), we choose $\delta_{\max }=\delta_{\min }=1, \mu(t)=0.89 e^{-3.5 t}+0.01$. The simulation results are shown in Figures 4 and 5. From Figures 4 and 5 , we know that the present control method can guarantee that all the variables are bounded. Moreover, the error $e(t)$ remains within the prescribed performance bounds for all time. All the aforementioned results clearly show that the present PPF-based control method (18) can obtain better regulation performance; that is, $e(t)$ can be retained within the PPF bound and achieves faster convergence performance compared to method (38).

The simulation results show that the proposed prescribed transient and steady-state performances are achieved. Thus, the numerical simulations verify theoretical analysis.

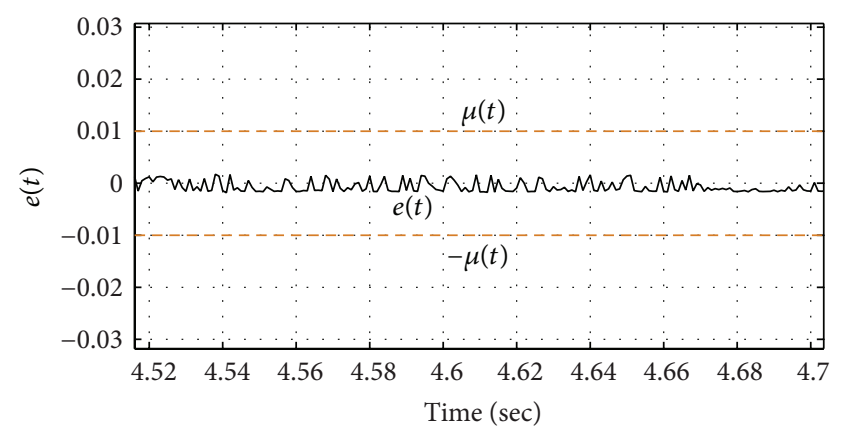

Figure 5: Time response $e(t)(t \in[4.52,4.70])$ of error dynamic system (36) under the present method of (18).

\section{Conclusions}

For a class of uncertain HPS with unknown control gain, the adaptive fuzzy feedback tracking control problem has been considered. By using prescribed performance functions, we transform the system into an equivalent one, and the fuzzy logic systems are used to identify the unknown nonlinear functions. It is sufficient to guarantee the boundedness of all the variables in the closed-loop system. Simulation results have shown the effectiveness of the proposed scheme.

\section{Conflict of Interests}

The authors declare that there is no conflict of interests regarding the publication of this paper.

\section{Acknowledgments}

The authors gratefully acknowledge the support of the National Natural Science Foundation of China (61403157), the Natural Science Foundation of Anhui Province (1508085 QA16), Anhui Province University Humanities and Social Science Research Base project (SK2015A158, SK2015A159), the Scientific Research Project of Huainan Normal University (2014xj07zd, 2015xj07zd), and the Natural Science Foundation for the Higher Education Institutions of Anhui Province of China (KJ2015A256).

\section{References}

[1] D. X. Yang and J. L. Zhou, "Connections among several chaos feedback control approaches and chaotic vibration control of mechanical systems," Communications in Nonlinear Science and Numerical Simulation, vol. 19, no. 11, pp. 3954-3968, 2014.

[2] Y. Chen, X. Wu, and Z. Liu, "Global chaos synchronization of electro-mechanical gyrostat systems via variable substitution control," Chaos, Solitons and Fractals, vol. 42, no. 2, pp. 11971205, 2009.

[3] Y.-J. Sun, "A novel chaos synchronization of uncertain mechanical systems with parameter mismatchings, external excitations, and chaotic vibrations," Communications in Nonlinear Science and Numerical Simulation, vol. 17, no. 2, pp. 496-504, 2012.

[4] X. F. Wu, J. P. Cai, and M. H. Wang, "Master-slave chaos synchronization criteria for the horizontal platform systems 
via linear state error feedback control," Journal of Sound and Vibration, vol. 295, no. 1-2, pp. 378-387, 2006.

[5] N.-S. Pai and S.-P. Chang, "Design and implementation of fuzzy sliding mode controllers for generalized projective synchronization of chaos horizontal platform systems," Computers and Mathematics with Applications, vol. 64, no. 5, pp. 709-720, 2012.

[6] K. Ding and Q.-L. Han, "Master-slave synchronization criteria for horizontal platform systems using time delay feedback control," Journal of Sound and Vibration, vol. 330, no. 11, pp. 2419-2436, 2011.

[7] X. F. Wu, J. P. Cai, and M. H. Wang, "Robust synchronization of chaotic horizontal platform systems with phase difference," Journal of Sound and Vibration, vol. 305, no. 3, pp. 481-491, 2007.

[8] N.-S. Pai and H.-T. Yau, "Suppression of chaotic behavior in horizontal platform systems based on an adaptive sliding mode control scheme," Communications in Nonlinear Science and Numerical Simulation, vol. 16, no. 1, pp. 133-143, 2011.

[9] M. P. Aghababa and H. P. Aghababa, "Synchronization of mechanical horizontal platform systems in finite time," Applied Mathematical Modelling, vol. 36, no. 10, pp. 4579-4591, 2012.

[10] W. Xiang and X. J. Liu, "An adaptive fuzzy sliding mode control design for a class of uncertain horizontal platform systems," Abstract and Applied Analysis, vol. 2014, Article ID 154383, 6 pages, 2014.

[11] L. Long and J. Zhao, "Adaptive fuzzy tracking control of switched uncertain nonlinear systems with unstable subsystems," Fuzzy Sets and Systems, vol. 273, no. 15, pp. 49-67, 2015.

[12] S. Labiod, M. S. Boucherit, and T. M. Guerra, "Adaptive fuzzy control of a class of MIMO nonlinear systems," Fuzzy Sets and Systems, vol. 151, no. 1, pp. 59-77, 2005.

[13] T. P. Zhang and X. N. Xia, "Decentralized adaptive fuzzy output feedback control of stochastic nonlinear large-scale systems with dynamic uncertainties,' Information Sciences, vol. 315, no. 10, pp. 17-38, 2015.

[14] H. X. Zhang, F. Shi, and Y. T. Liu, "Enhancing optimal excitation control by adaptive fuzzy logic rules," International Journal of Electrical Power and Energy Systems, vol. 63, pp. 226-235, 2014.

[15] A. Senouci and A. Boukabou, "Predictive control and synchronization of chaotic and hyperchaotic systems based on a T-S fuzzy model," Mathematics and Computers in Simulation, vol. 105, pp. 62-78, 2014.

[16] C. P. Bechlioulis and G. A. Rovithakis, "Robust adaptive control of feedback linearizable MIMO nonlinear systems with prescribed performance," IEEE Transactions on Automatic Control, vol. 53, no. 9, pp. 2090-2099, 2008.

[17] J. Na, Q. Chen, X. M. Ren, and Y. Guo, "Adaptive prescribed performance motion control of servo mechanisms with friction compensation," IEEE Transactions on Industrial Electronics, vol. 61, no. 1, pp. 486-494, 2014.

[18] Y. G. Sun and H. Liu, "Fuzzy adaptive prescribed performance control for MIMO uncertain chaotic systems in nonstrict feedback form," Discrete Dynamics in Nature and Society, vol. 2014, Article ID 192387, 6 pages, 2014. 


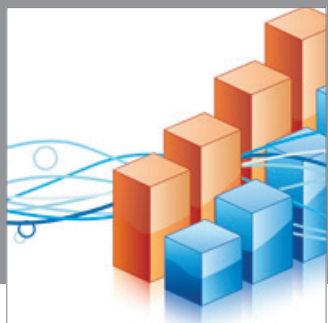

Advances in

Operations Research

mansans

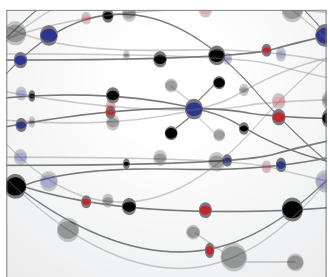

The Scientific World Journal
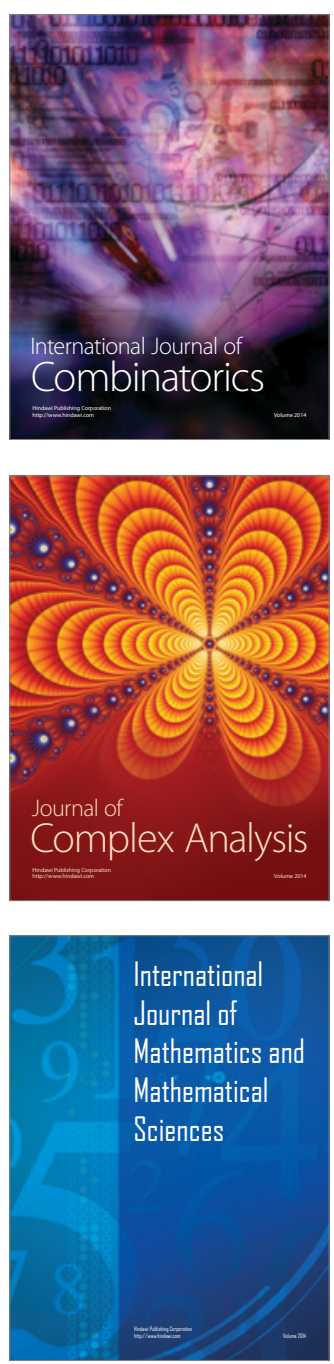
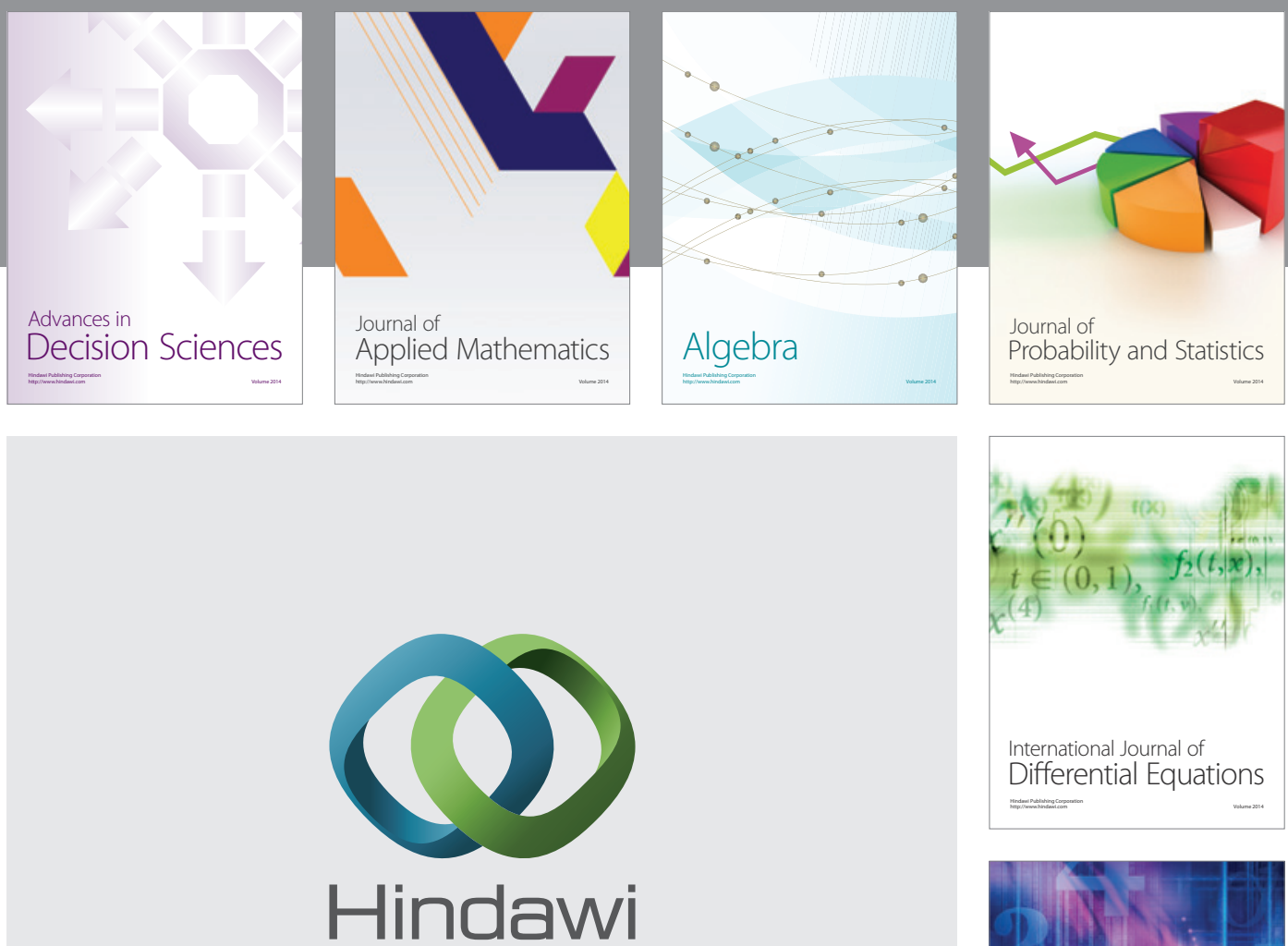

Submit your manuscripts at http://www.hindawi.com
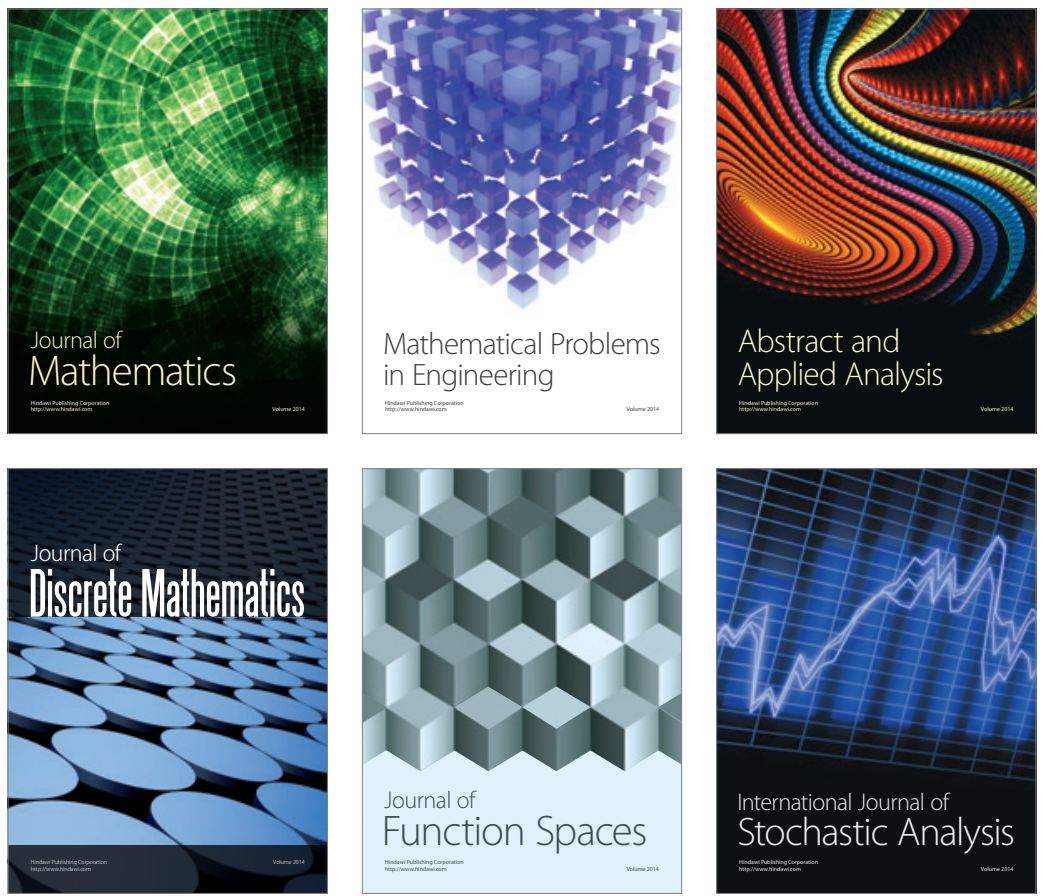

Journal of

Function Spaces

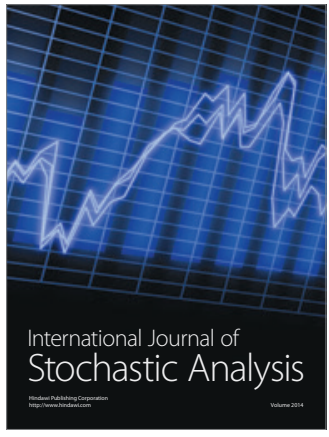

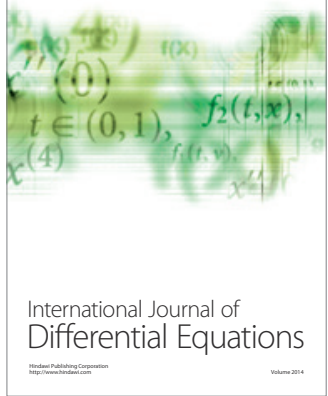
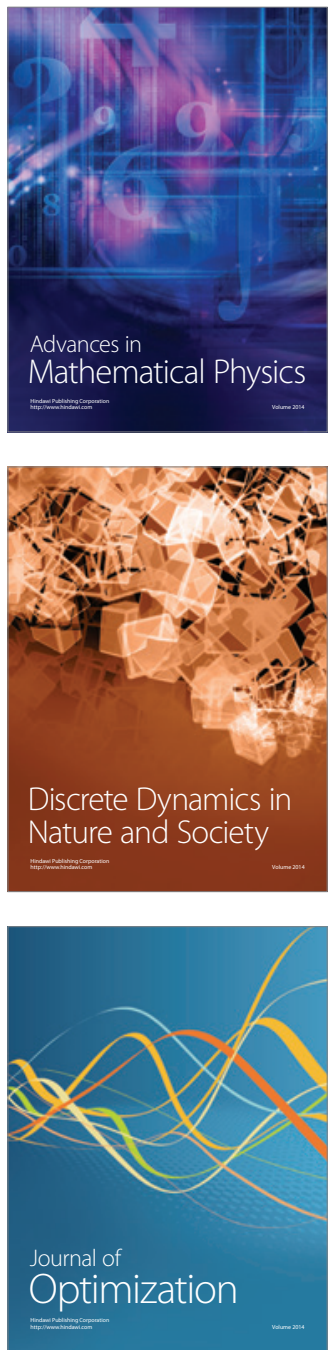